02

\title{
Экспериментальное наблюдение $s$-компоненты сверхпроводящего спаривания в тонких неупорядоченных пленках ВТСП на основе YBCO
}

\author{
(C) А.В. Антонов ${ }^{1}$, А.И. Елькина ${ }^{1}$, В.К. Васильев ${ }^{1}$, М.А. Галин ${ }^{1}$, Д.В. Мастеров ${ }^{1}$, А.Н. Михайлов ${ }^{2}$, \\ C.В. Морозов ${ }^{1}$, С.А. Павлов ${ }^{1}$, А.Е. Парафин ${ }^{1,2}$, Д.И. Тетельбаум ${ }^{2}$, \\ С.С. Уставщиков ${ }^{1,2}$, П.А. Юнин ${ }^{1,2}$, Д.А. Савинов ${ }^{1,2, \uparrow}$ \\ ${ }^{1}$ Институт фоизики микроструктур РАН, \\ Нижний Новгород, Россия \\ ${ }^{2}$ Нижегородский государственный университет им. Н.И. Лобачевского, \\ Нижний Новгород, Россия \\ ฯ E-mail: savinovda@ipmras.ru
}

Поступила в Редакцию 26 марта 2020 г.

В окончательной редакции 26 марта 2020 г.

Принята к публикации 2 апреля 2020 г.

\begin{abstract}
Представлены оригинальные результаты исследования тонких неупорядоченных пленок ВТСП на основе YВСО. Выполнено несколько экспериментов, которые подтверждают теоретическое предсказание возможного формирования $s$-фазы сверхпроводящего спаривания в неупорядоченных сверхпроводниках $d$-типа при постепенном уменьшении средней длины свободного пробега. В качестве образцов использованы пленки $\mathrm{YBa}_{2} \mathrm{Cu}_{3} \mathrm{O}_{7-x}$, в которых экспериментально подтвержден $d$-тип сверхпроводящего состояния. Постепенное уменьшение длины свободного пробега достигалось как с помощью термического отжига, так и в результате ионного облучения. Эксперименты включали измерения температурной зависимости лондоновской глубины проникновения $\lambda$ и резистивные исследования.
\end{abstract}

Ключевые слова:высокотемпературные сверхпроводники, тонкие пленки, беспорядок, ионное облучение, термический отжиг, лондоновская длина, резистивные измерения.

DOI: 10.21883/FTT.2020.09.49766.33H

\section{1. Введение}

В последние несколько лет наблюдается яркий всплеск в исследованиях высокотемпературных сверхпроводников (ВТСП) с высокой степенью неупорядоченности (см., например, работы [1-4]). Интерес к ним обусловлен тем, что вблизи перехода „сверхпроводникнормальный металл“ (или „сверхпроводник-изолятор“) возможно проявление достаточно необычных свойств таких материалов. В частности, экспериментально обнаружено, что электронный транспорт в несверхпроводящем состоянии пленок ҮВСО, содержащих решетку наноотверстий, осуществляется не отдельными электронами, а куперовскими парами [1]. Также необычной особенностью неупорядоченных ВТСП материалов является возможность формирования $s$-фазы сверхпроводящего спаривания при постепенном увеличении степени беспорядка в образцах, что было недавно предсказано теоретически [2,3]. Более того, свойства таких материалов во внешнем магнитном поле $H$ оказываются еще более загадочными - температурная зависимость верхнего критического поля $H_{c 2}(T)$ оказывается монотонно возрастающей, что приводит к возможности стимулирования сверхпроводимости с помощью приложения $H$ [4], тогда как обычно внешнее магнитное поле всегда подавляет критическую температуру $T_{c}$. Некоторые другие аномальные особенности фазовых диаграмм на плоскости внешнее магнитное поле $H$ - температура $T$ были исследованы экспериментально в наших недавних работах для тонких пленок $\mathrm{YBa}_{2} \mathrm{Cu}_{3} \mathrm{O}_{7-x}$ с разной степенью неупорядоченности $[5,6]$. Неупорядоченность в образцах менялась постепенно с помощью метода ионного облучения. Было проведено последовательно несколько актов ионного облучения. Используя расчет по программе SRIM [7], была оценена типичная концентрация дефектов в образце на каждой стадии ионной имплантации. Согласно данным оценкам, мы не смогли вплотную приблизиться к переходу „сверхпроводник-изолятор“. Однако, даже в этом случае обнаружены некоторые интересные особенности. В частности, для каждой дозы ионного облучения $n_{D}$ был изучен характер зависимостей $H_{c 2}(T)$, рассчитанных по заданному постоянному резистивному уровню. Особое внимание было уделено началу сверхпроводящего перехода, а именно, особенностям кривых $H_{c 2}(T)$, в которых верхнее критическое магнитное поле $H_{c 2}$ было определено, как значение $H$, соответствующее достаточно высоким сопротивлениям внутри сверхпроводящего перехода - порядка 90\% от максимального сопротивления на переходе. Обнаружено, что при постепенном увеличении дозы ионной имплантации наблюдается необычное уменьшение наклона верхнего критического 
поля вблизи $T_{c 0}=T_{c}(H=0)$, тогда как обычно рост концентрации дефектов приводит к увеличению локального наклона линии фазового перехода $H_{c 2}(T)$. Также было обнаружено, что температурная зависимость верхнего критического поля обладает положительной кривизной вблизи $T_{c 0}$. В рамках теоретической модели, для определения критической температуры $T_{c}$ первых зародышей сверхпроводящей фазы, возникающих в разупорядоченном образце при постепенном понижении температуры во внешнем магнитном поле $H$, была применена обобщенная теория Гинзбурга-Ландау. Обобщение заключалось в учете пространственной зависимости коэффициента диффузии, а также в учете зависимости критической температуры $T_{c 0}$ от концентрации нейтральных дефектов в образце. В итоге, указанные особенности линии фазового перехода были описаны теоретически с помощью простых формул (детали см. в работах $[5,6])$.

Настоящая работа посвящена продолжению экспериментальных исследований неупорядоченных пленок $\mathrm{YBa}_{2} \mathrm{Cu}_{3} \mathrm{O}_{7-x}$ (использованы большие дозы ионного облучения), а также содержит новые результаты, позволяющие осуществить экспериментальную проверку теоретического предсказания работ [2,3] о возможном формировании $s$-фазы параметра порядка в сильно неупорядоченных сверхпроводниках $d$-типа, когда существенны пространственные флуктуации средней длины свободного пробега. Фактически, в таких системах возникает джозефсоновская сеть гранул с $s$-типом сверхпроводящего спаривания, которые обладают некоторым распределением наведенного параметра порядка (каждая гранула характеризуется определенным значением $T_{c}$ и определенной фазой параметра порядка). В результате поведение системы напоминает поведение спиновых стекол. Это означает, что при $T \rightarrow 0$ температурная зависимость лондоновской глубины проникновения $\lambda$ должна иметь термоактивационный характер, подобно тому, как это происходит в обычных сверхпроводниках $s$-типа. Данное предположение было подтверждено экспериментально в настоящей работе, а именно было выполнено два различных исследования. В эксперименте № 1 мы изучали температурную зависимость лондоновской глубины проникновения $\lambda$ с помощью методики - „две катушки“. В рамках эксперимента № 2 мы провели резистивные измерения узких мостиков для разных степеней беспорядка и изучили зависимость $T_{c}\left(n_{D}\right)$ для разных уровней сопротивления внутри сверхпроводящего перехода. Таким образом, мы сравнили поведение экспериментальных зависимостей $\lambda(T)$ и $T_{c}\left(n_{D}\right)$ с известными теоретическими формулами для сверхпроводников с $s$-типом спаривания. В итоге, было найдено хорошее соответствие экспериментальных данных и теоретических результатов для образцов с высокой степенью неупорядоченности.

\section{2. Изготовление образцов и детали эксперимента}

Пленки $\mathrm{YBaCuO}$ выращивались методом магнетронного напыления на подложке из алюмината лантана LaAlO. В экспериментах использовались пленки с толщинами $d=4 \mathrm{~nm}$ и $200 \mathrm{~nm}$. Для экспериментального исследования № 1 мы использовали ультратонкую пленку с толщиной $d=4 \mathrm{~nm}$. Выбор столь малой толщины обусловлен сильной экранировкой магнитного поля, создаваемого одной катушкой, в результате чего магнитное поле, принимаемое второй катушкой, оказывается сильно подавленным при больших толщинах пленки. Это накладывает жесткие условия на толщину ввиду проблемы выделения полезного сигнала на фоне шумов. Беспорядок в данной пленке создавался с помощью последовательных актов термического отжига, в результате чего критическая температура $T_{c 0}$ постепенно понижалась.

Для эксперимента № 2 была выбрана пленка с $d=200 \mathrm{~nm}$. Для модификации свойств пленки мы использовали облучение ионами кислорода $\mathrm{O}^{+}$. Облучение использовалось дважды: для формирования рисунка сверхпроводящих тестовых мостиков и для организации беспорядка в образце. Для достижения первой цели облучение проводилось с использованием ионов с энергией $120 \mathrm{keV}$ и дозой $2 \cdot 10^{15} \mathrm{~cm}^{-2}$ через фоторезистивную маску толщиной $1.4 \mu \mathrm{m}$. В результате был сформирован рисунок мостиков с различными ширинами, но одинаковым соотношением длина/ширина, равным 5/1 для каждого мостика. В рамках данной работы мы провели серию последовательных транспортных измерений (после каждого из актов ионной имплантации) для одного из мостиков, ширина которого составила $50 \mu \mathrm{m}$. Контактные площадки были изготовлены методом термического напыления серебра через металлическую маску. Толщина слоя серебра равнялась $100 \mu \mathrm{m}$. После имплантации фоторезист удалялся в ацетоне. Критическая температура и плотность критического тока пленки $\mathrm{YBaCuO}$, измеренные на мостиках, составили, соответственно, $T_{c 0}=91 \mathrm{~K}$ и $J_{c}=4 \cdot 10^{6} \mathrm{~A} \cdot \mathrm{cm}^{-2}$ при $T=77 \mathrm{~K}$, независимо от ширины мостика. Удельное сопротивление каждого из мостиков при температуре $T=100 \mathrm{~K}$ составило величину $100 \mu \Omega \cdot \mathrm{cm}$, что соответствует оптимуму кислородного допирования пленки при эпитаксии, т.е. значению $x=0.1$ в пленке $\mathrm{YBa}_{2} \mathrm{Cu}_{3} \mathrm{O}_{7-x}$. Для модуляции характеристик беспорядка в мостиках было использовано ионное облучение без фоторезистивной маски, которое проводилось при комнатной температуре с энергией ионов $100 \mathrm{keV}$. В рамках эксперимента № 2 было проведено 6 последовательных актов ионного облучения так, что накопленные дозы $n_{D}$ после каждого из актов составили $3 \cdot 10^{13} \mathrm{~cm}^{-2}, 4.3 \cdot 10^{13} \mathrm{~cm}^{-2}, 6 \cdot 10^{13} \mathrm{~cm}^{-2}$, $6.3 \cdot 10^{13} \mathrm{~cm}^{-2}, 6.6 \cdot 10^{13} \mathrm{~cm}^{-2}, 7.5 \cdot 10^{13} \mathrm{~cm}^{-2}$ соответственно. Сопротивление образца измерялось стандартным четырехзондовым методом. 

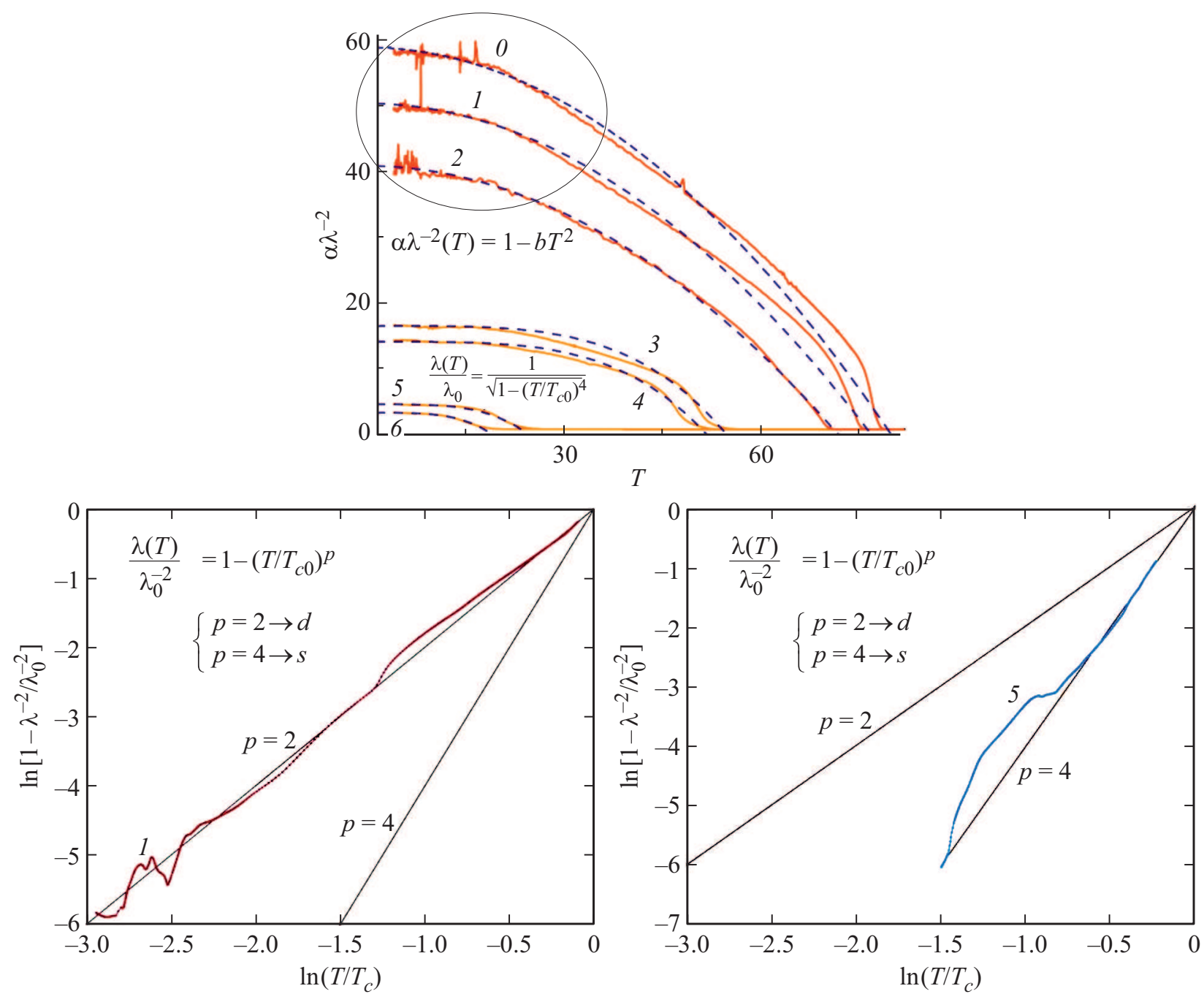

Рис. 1. Зависимости $\lambda^{-2}(T)$ для пленки толщиной $d=4 \mathrm{~nm}$ и разных стадий термического отжига. Здесь $\alpha$ и $b-$ постоянные коэффициенты. Сплошными линиями изображены экспериментальные данные, пунктирные линии соответствуют представленным на рисунке формульным аппроксимациям. Овалом обозначена температурная область наилучшей аппроксимации степенной функцией для исходного образца, а также для случаев первой и второй стадий термического отжига. Цифрами обозначены номера актов термического отжига. Внизу представлены зависимости (1) и (5), а также выбранные две аппроксимирующие функции в билогарифмическом масштабе.

\section{3. Результаты экспериментов}

С помощью методики - „две катушки“ (эксперимент № 1) мы измерили температурную зависимость $\lambda$ для образца (пленка толщиной $d=4 \mathrm{~nm}$ ) с разной степенью неупорядоченности. Для исходного образца данная зависимость оказалась степенной при низких температурах (см. рис. 1), как это обычно происходит в сверхпроводниках с необычным типом спаривания (в данном случае с $d$-типом спаривания). Действительно, в случае нестандартного спаривания энергетическая щель в спектре квазичастичных возбуждений обращается в нуль вдоль некоторых линий на поверхности Ферми, и возникает степенная зависимость $\lambda(T)$ [8]. При последующих измерениях $\lambda(T)$ пленка предварительно подвергалась 6 последовательным актам термического отжига. Возникающий таким образом в пленке беспорядок приводил к постепенному понижению критической температуры $T_{c 0}$, что явно продемонстрировано на рис. 1 - при увеличении количества актов проведенных отжигов кривая $\lambda(T)$ сдвигается в область меньших температур и отсекает на оси абсцисс все меньшее значение температуры, которое соответствует критической температуре $T_{c 0}$. Выяснено, что для $T_{c 0}$, превышающих $50 \mathrm{~K}$, качественный вид $\lambda(T)$ практически не меняется (см. рис. 1). Однако, для $T_{c 0}$ порядка $50 \mathrm{~K}$ и ниже температурная зависимость лондоновской глубины проникновения имеет ярко выраженный активационный характер при $T \rightarrow 0-$ подобно тому, как это происходит в рамках модели БКШ: $\lambda(T) \propto \exp \left(-\Delta_{0} / T\right)$. Форма зависимости $\lambda(T)$ во всем температурном интервале для сверхпроводников со стандартным спариванием хорошо 


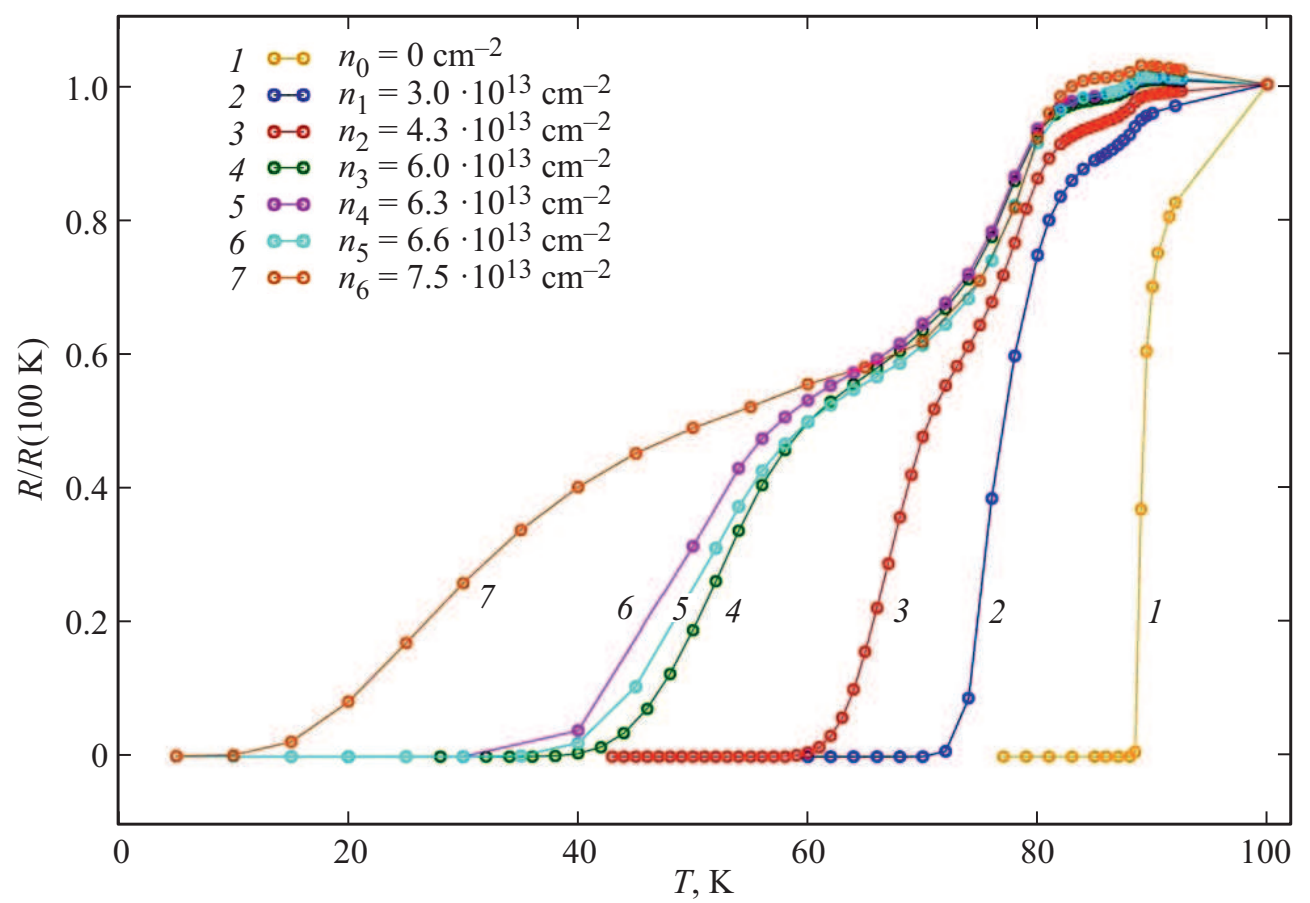

Рис. 2. Зависимости нормированного сопротивления мостика $R / R(100 \mathrm{~K})$ от температуры $T$, соответствующие различным дозам ионного облучения $n_{D}$ (значения доз $n_{D}$ указаны на рисунке).

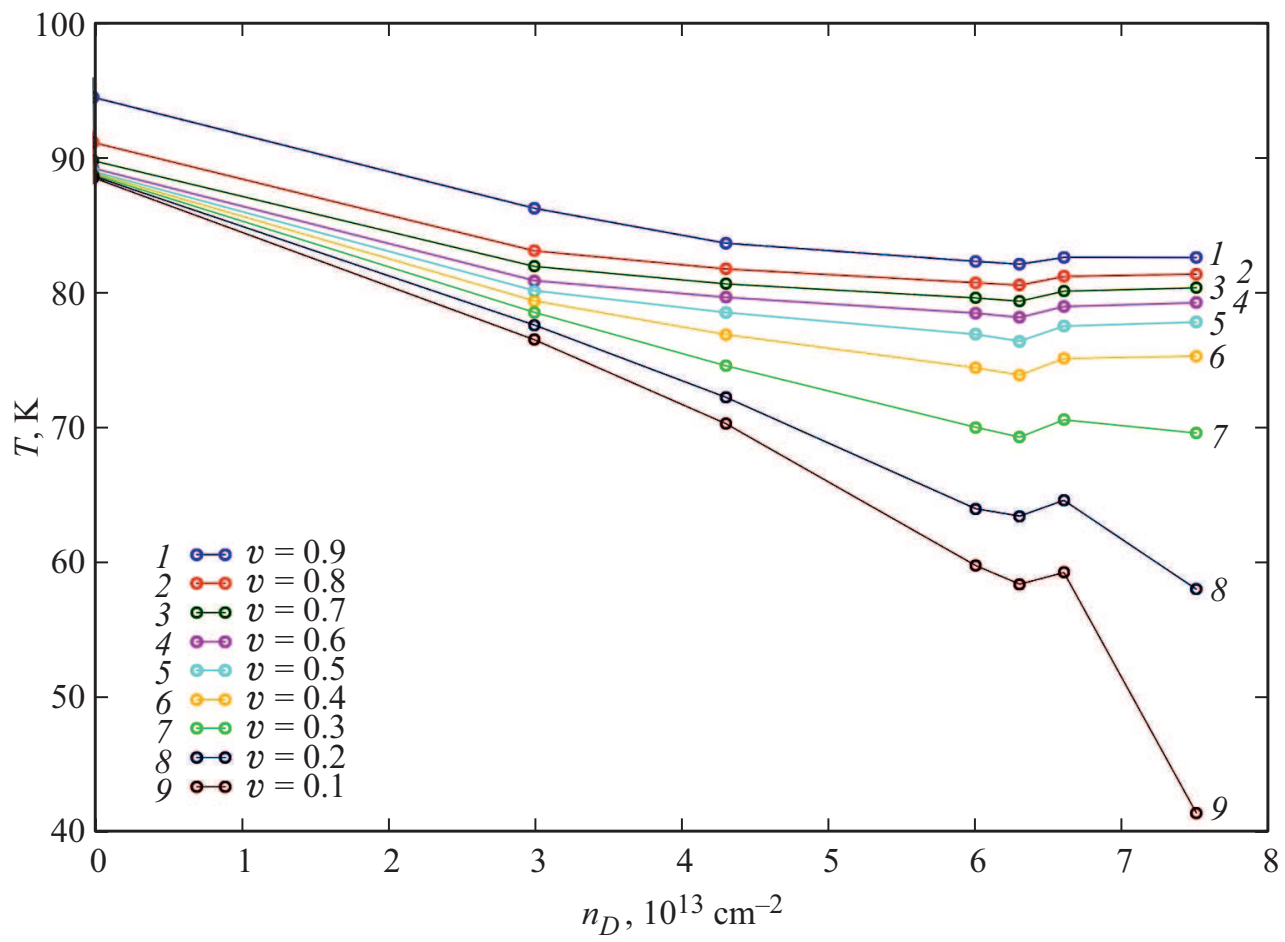

Рис. 3. Зависимости критической температуры $T_{c 0}$ от дозы ионного облучения $n_{D}$, рассчитанные для разных резистивных уровней профилей $R(T)$, представленных на рис. 2, в долях относительно сопротивления при $T=100 \mathrm{~K}$ (указанные доли представлены на рисунке).

описывается функцией $\lambda(T) / \lambda_{0}=\left[1-\left(T / T_{c 0}\right)^{4}\right]^{-1 / 2}$. Как показано на рис. 1, полученные экспериментальные данные для 3-ей и последующих стадий термического отжига (зависимости, для которых $T_{c 0}$ ниже $50 \mathrm{~K}$ ) хорошо согласуются с данной формулой. В нижней части рисунка отдельно изображены зависимости (1) и (5), а 
также выбранные две аппроксимирующие функции в билогарифмическом масштабе. Видно, что для кривой (1) больше подходит степенная аппроксимация с показателем $p=2$, а для кривой $(5)-$ с показателем $p=4$ (см. рис. 1). Данные графики показывают, что кривые, соответствующие слабо разупорядоченным пленкам, лучше описываются степенным законом с показателем $p=2$, а сильно неупорядоченным пленкам - степенным законом с показателем $p=4$. Полученные результаты могут являться свидетельством формирования $s$-компоненты спаривания в пленке $\mathrm{YBa}_{2} \mathrm{Cu}_{3} \mathrm{O}_{7-x}$ при постепенном увеличении степени беспорядка в ней.

В рамках эксперимента № 2 проведены транспортные измерения разупорядоченных мостиков, сформированных, на основе пленок $\mathrm{YBa}_{2} \mathrm{Cu}_{3} \mathrm{O}_{7-x}$ с толщиной $d=200 \mathrm{~nm}$. Как уже было отмечено, разупорядоченность достигалась постепенно - с помощью последовательных актов облучения ионами кислорода. Измерения проводились после каждого акта. Таким способом мы проанализировали особенности сверхпроводящего фазового перехода для разных степеней беспорядка в системе (см. рис. 2). Зависимость критической температуры $T_{c 0}$ от дозы имплантированных ионов $n_{D}-$ фактически, от концентрации нейтральных дефектов, возникших в образце, представлена на рис. 3. Данная зависимость определялась из рис. 2 по разным резистивным уровням, соответствующим определенной доле сопротивления относительно сопротивления на начале сверхпроводящего перехода. Под началом сверхпроводящего перехода подразумевается значение критической температуры, ниже которой сопротивление исходного (без облучения) образца начинает резко понижаться. Это значение соответствует $100 \mathrm{~K}$. Особое внимание было уделено исследованию зависимостей $T_{c 0}\left(n_{D}\right)$ для достаточно высоких резистивных уровней сверхпроводящего перехода, когда в образце возникает сеть случайно распределенных сверхпроводящих островков с $d$-типом спаривания внутри материала, находящегося преимущественно в нормальном состоянии, обусловленного ионным облучением. Согласно эффекту близости, аномальная функция Грина $F$ каждого островка экспоненциально спадает через границу сверхпроводник/нормальный металл и, таким образом, имеет ненулевую область определения в окружающем данный островок нормальном „фоне“. Однако, она уже не может обладать симметрией строго $d$-типа, поскольку в данной области присутствует сильная разупорядоченность кристаллической решетки из-за ионного облучения. Поэтому функция $F$ представляет собой суперпозицию функций, соответствующих разным симметриям параметра порядка $-s, d, p$ и т.д. Однако, ввиду того, что необычная сверхпроводимость (все кроме $s$-типа) сильно подавляется введением нейтральных примесей, наиболее дальнодействующим эффектом обладает $F$-функция с $s$-типом спаривания. Фактически, в таких системах возникает джозефсоновская сеть гранул c $s$-типом сверхпроводящего спаривания, которые обладают некоторым распределением наведенного параметра порядка (каждая гранула характеризуется определенным значением $T_{c 0}$ и определенной фазой параметра порядка), а в системе глобально должна возникать сверхпроводимость $s$-типа (см. работы $[2,3]$ ). Действительно, как видно из рис. 3 , для доз $n_{D}=5 \cdot 10^{13} \mathrm{~cm}^{-2}$ и выше критическая температура $T_{c 0}$ практически не зависит от $n_{D}$, как это обычно происходит в сверхпроводниках $s$-типа - их критическая температура обычно не зависит от концентрации нейтральных дефектов вплоть до перехода „сверхпроводник-изолятор“.

\section{4. Заключение}

В данной работе изучено возможное влияние беспорядка в тонких эпитаксиальных пленках ВТСП $\mathrm{YBa}_{2} \mathrm{Cu}_{3} \mathrm{O}_{7-x}$ на смену типа спаривания в образцах с $d$-волнового на $s$-волновой. Проведено два различных эксперимента, в результате которых исследованы зависимость лондоновской глубины проникновения от температуры, а также зависимость критической температуры от концентрации дефектов в образце. Данные экспериментальные зависимости найдены для разных степеней беспорядка в системе. Организация беспорядка производилась в результате термического отжига и методом ионного облучения. В результате, вблизи перехода „сверхпроводник-изолятор“ удалось получить хорошее соответствие экспериментальных зависимостей и известных теоретических формул, справедливых для $s$-волновых сверхпроводников. Таким образом, в данной работе представлено экспериментальное подтверждение необычного теоретического предсказания работ [2,3] о возможном формировании $s$-типа сверхпроводимости в $d$-сверхпроводниках в грязном пределе, когда существенны флуктуации коэффициента диффузии в системе.

\section{Благодарности}

Авторы выражают благодарность А.С. Мельникову за многократное обсуждение результатов работы и ценные советы. Авторы также благодарны В.В. Курину, В.И. Гавриленко и С.В. Шарову за полезные замечания.

\section{Финансирование работы}

Настоящая работа выполнена при поддержке гранта РФФИ № 18-42-520051. Работа также поддержана программой Президиума РАН I.1 „Фундаментальные проблемы высокотемпературной сверхпроводимости“ и выполнена в рамках государственного задания ИФМ РАН за 2020 год. В работе использовано оборудование ЦКП „Физика и технология микро- и наноструктур“.

\section{Конфликт интересов}

Авторы заявляют, что у них нет конфликта интересов. 


\section{Список литературы}

[1] C. Yang et al. Science 10.1126, 5798 (2019).

[2] A. Keles, A.V. Andreev, B.Z. Spivak, S.A. Kivelson. JETP, 119, 1109 (2014)

[3] S.A. Kivelson, B. Spivak. Phys. Rev. B 92, 184502 (2015).

[4] M. Schiulaz, C.L. Baldwin, C.R. Laumann, B.Z. Spivak. Phys. Rev. B 98, 094508 (2018).

[5] А.В. Антонов, А.В. Иконников, Д.В. Мастеров, А.Н. Михайлов, С.В. Морозов, Ю.Н. Ноздрин, С.А. Павлов, А.Е. Парафин, Д.И. Тетельбаум, С.С. Уставщиков, П.А. Юнин, Д.А. Савинов. ФТТ 61, 1573 (2019).

[6] A.V. Antonov, A.V. Ikonnikov, D.V. Masterov, A.N. Mikhailov, S.V. Morozov, Yu.N. Nozdrin, S.A. Pavlov, A.E. Parafin, D.I. Tetel'baum, S.S. Ustavschikov, V.K. Vasiliev, P.A. Yunin, D.A. Savinov. Physica C, 568, 1353581 (2020).

[7] J.F. Zeigler, J.P. Biersack, M.D. Zeigler. The Stopping and Range of Ions in Matter (SRIM). USA Chester Maryland (2008).

[8] Н.М. Плакида, Высокотемпературные сверхпроводники. Международная программа образования, Москва (1996).

Редактор К.В. Емщев 